\title{
Multireference Ground and Excited State Electronic Structures of Free- versus Iron Porphyrin-Carbenes
}

\author{
Gautam D. Stroscio ${ }^{\mathrm{a}}$, Martin Srnec ${ }^{\mathrm{b}, *}$, and Ryan G. Hadt ${ }^{\mathrm{a}, *}$
}

aDivision of Chemistry and Chemical Engineering, Arthur Amos Noyes Laboratory of Chemical Physics, California Institute of Technology, Pasadena, California 91125, United States

bJ. Heyrovský Institute of Physical Chemistry, Czech Academy of Sciences, Prague 8, 18223, Czech Republic

Corresponding Authors: martin.srnec@jh-inst.cas.cz, rghadt@,caltech.edu

\begin{abstract}
Iron porphyrin carbenes (IPCs) are important reaction intermediates in engineered carbene transferase enzymes and homogeneous catalysis. However, discrepancies between theory and experiment complicate the understanding of IPC electronic structure (i.e., open- vs. closed-shell singlet (OSS vs. CSS)). Here we investigate the structurally dependent ground and excited spin state energetics of a free carbene and its IPC analogs. Only multireference $a b$ initio wave function

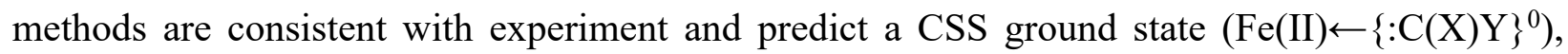
contrary to density functional theory (DFT). The OSS is a high-lying metal-to-ligand charge transfer (MLCT) excited state that is sensitive to the nature of the axial ligand. Furthermore, potential energy surfaces (PESs) along the Fe-C bond elongation coordinate exhibit strong mixings between CSS/OSS characters, which can be an important feature for describing reaction mechanisms. Future studies on IPC reaction coordinates should evaluate contributions from ground and excited state multireference character.
\end{abstract}


Iron porphyrin carbenes (IPCs) carry out a variety of important chemistries, including $\mathrm{B}-\mathrm{H},{ }^{[1,2]} \mathrm{C}-$ $\mathrm{H},{ }^{[3]} \mathrm{N}-\mathrm{H},{ }^{[4,5]} \mathrm{O}-\mathrm{H},{ }^{[6]} \mathrm{Si}-\mathrm{H},{ }^{[7,8]}$ and $\mathrm{S}-\mathrm{H}^{[9,10]}$ insertion, as well as cyclopropanation, ${ }^{[11-15]}$ cyclopropenation, ${ }^{[14]}$ and carbonyl olefination. ${ }^{[16]}$ This diverse reactivity has spurred interest in defining the electron structures of IPCs. Relevant descriptions include: $\mathrm{Fe}(\mathrm{IV})=\{\mathrm{C}(\mathrm{X}) \mathrm{Y}\}^{2-}$, $\mathrm{Fe}(\mathrm{II}) \leftarrow\{: \mathrm{C}(\mathrm{X}) \mathrm{Y}\}$, or $\mathrm{Fe}(\mathrm{III})-\{\mathrm{C}(\mathrm{X}) \mathrm{Y}\}^{-\bullet}$, with $\mathrm{X}$ and $\mathrm{Y}$ corresponding to different chemical groups. In $\mathrm{Fe}(\mathrm{III})$, the metal- and carbene-based electron spins can couple ferro- or antiferromagnetically to give the total spin states $S_{T}=1$ or 0 , respectively. The $F e(I I) \leftarrow\{: C(X) Y\}$ form is a closed shell singlet (CSS), differentiated from the antiferromagnetically coupled Fe(III)$\{\mathrm{C}(\mathrm{X}) \mathrm{Y}\}^{-\bullet}$ open shell singlet (OSS). Experimental data, including nuclear magnetic resonance $(\mathrm{NMR}){ }^{[17,18]}$ electron paramagnetic resonance (EPR) ${ }^{[8]}$ Mössbauer, ${ }^{[8,18,19]}$ and X-ray absorption spectroscopy (XAS) ${ }^{[19]}$ have largely been interpreted as reflecting the CSS Fe(II) state. However, studies utilizing density functional theory (DFT) have highlighted the potential importance of an OSS state. ${ }^{[8,20,21]}$ Here we study the structurally dependent energetics of the ground and excited state electronic structures of a free carbene and its IPC analogs. While DFT does not properly describe the OSS and its energetics due to its multiconfigurational character, multireference $a b$ initio methods provide a CSS ground state, more consistent with experimental observations. The OSS is predicted to be a metal-to-ligand charge transfer (MLCT) excited state $\sim 30-40 \mathrm{kcal} \mathrm{mol}^{-1}$ $\left(\sim 10500-14000 \mathrm{~cm}^{-1}\right)$ above the CSS ground state. Furthermore, the contributions of CSS/OSS character in the ground state evolve along the $\mathrm{Fe}-\mathrm{C}$ coordinate, which is important for evaluating transition metal carbene catalysis.

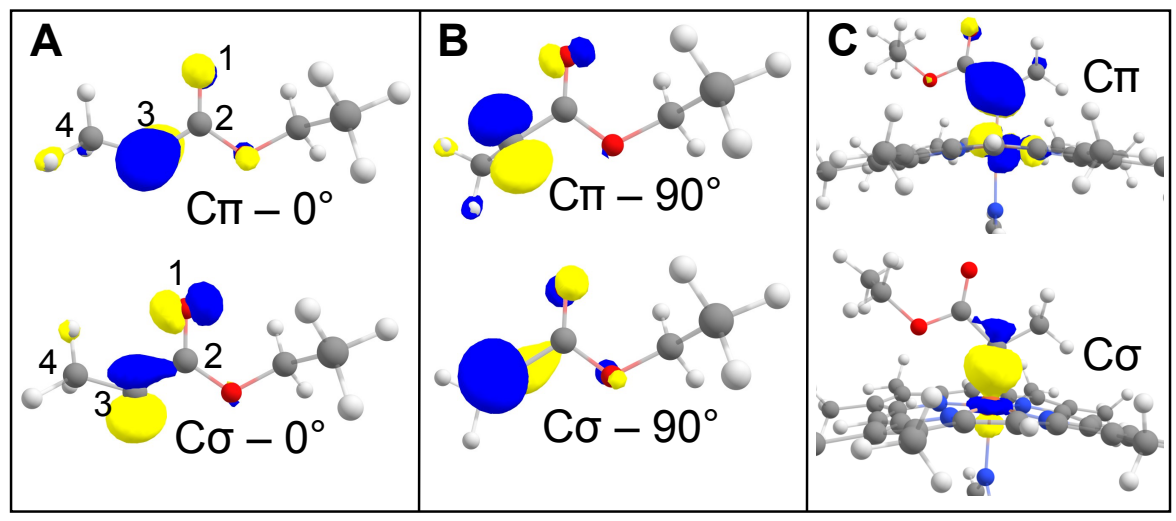

Figure 1. Free carbene $\mathrm{C} \sigma$ and $\mathrm{C} \pi$ orbitals for $(\mathrm{A}) 0^{\circ}$ and (B) $90^{\circ}$ conformations and (C) the analogous orbitals for the IPC. The dihedral angle of interest here is numbered in (A). 
The $\mathrm{C} \sigma$ - and $\mathrm{C} \pi$-type frontier molecular orbitals of the carbene are shown in Figure 1 . For the free carbene derived from ethyl 2-diazopropanate (Me-EDA), we have investigated the energetics of the states derived from the $(\mathrm{C} \sigma)^{2}(\mathrm{C} \pi)^{0}(\mathrm{CSS})$ and $(\mathrm{C} \sigma)^{1}(\mathrm{C} \pi)^{1}$ (OSS) electronic configurations. ${ }^{[22]}$ The DFT (BP86 and B3LYP) relaxed potential energy surfaces (PESs) from 0$180^{\circ}$ along the $\mathrm{O}-\mathrm{C}-\mathrm{C}-\mathrm{C}$ dihedral coordinate defined in Figure 1 are given in Figure 2A. Both functionals provide similar descriptions despite the $20 \%$ difference in Hartree-Fock exchange. At $0^{\circ}$ and $180^{\circ}$, the ground state is predicted to be a triplet (blue markers in Figure 2). The CSS (red markers in Figure 2) and OSS (green markers in Figure 2) are excited states $\sim 10-20 \mathrm{kcal} \mathrm{mol}^{-1}$ above the ground state. Upon rotating to $90^{\circ}$, the ground state becomes either the CSS or OSS, depending on the methodology. However, both states are within $\sim 2-3 \mathrm{kcal} \mathrm{mol}^{-1}$. Note the $360^{\circ}$ angular dependence is a mirror image about $180^{\circ}$ (Figure S1); thus only the $0-180^{\circ}$ scans are discussed here and below.

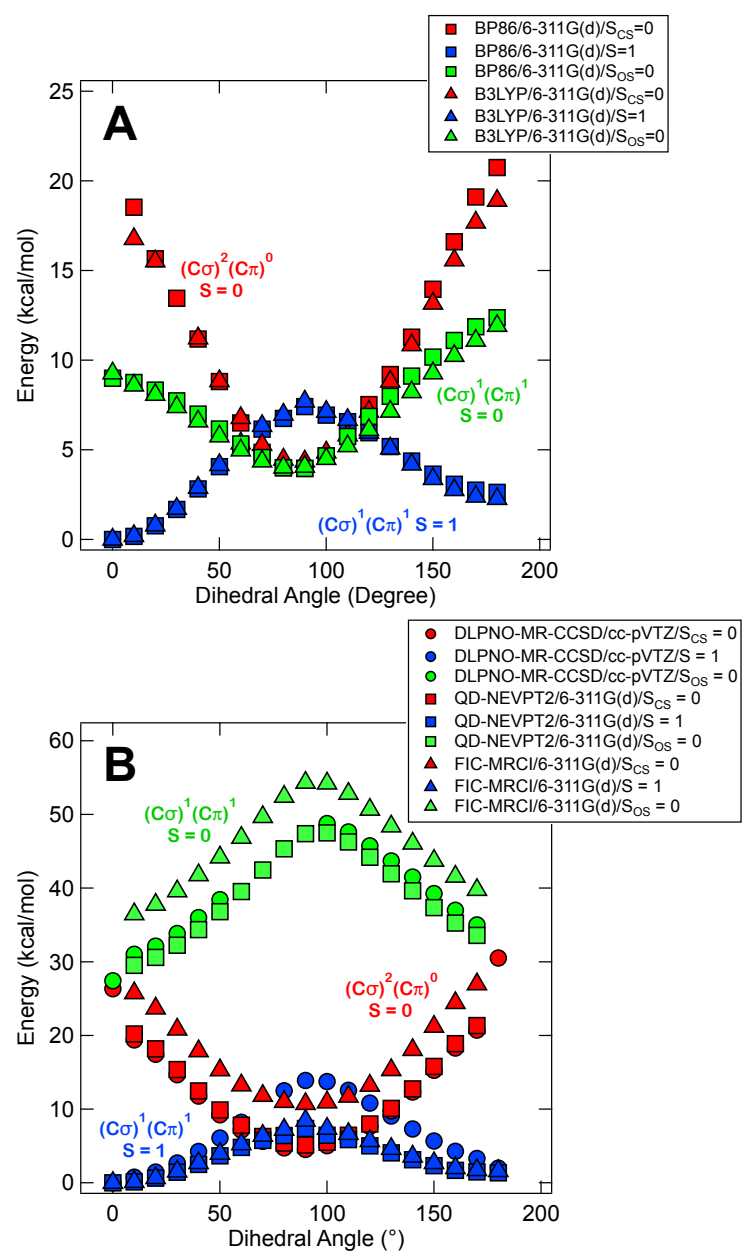

Figure 2. Ground and excited state PESs along the free carbene dihedral coordinate from Figure 1 using (A) DFT or (B) multireference ab initio methods. Acronyms for (B) are given in Supporting Information. 
PESs along the free carbene dihedral distortion for three multireference $a b$ initio methods as implemented in ORCA ${ }^{[23,24]}$ (DLPNO-MR-CSSD, FIC-MRCI, and QD-NEVPT2; acronyms and other computational details provided in Supporting Information) are presented in Figure 2B. In each case, an active space with two electrons in two $\mathrm{C} \sigma$ - and $\mathrm{C} \pi$-type orbitals was used (2-in-2 active space; orbitals in Figure 1). Similar to DFT, the triplet is the ground state at $0^{\circ} / 180^{\circ}$, and the CSS and OSS are excited states $\sim 25-30 \mathrm{kcal} \mathrm{mol}^{-1}$ above the ground state. However, at $90^{\circ}$, unlike DFT, the ground state is either a triplet or CSS, and the OSS is now the highest energy excited state considered here (green markers in Figure 2B). Additionally, the shape of the OSS manifold given by multireference methods is different than that of DFT. In particular, the OSS and triplet manifolds both have minima at $0^{\circ} / 180^{\circ}$ and maxima in the vicinity of $90^{\circ}$. The difference in energy between these states is simply $2 \mathrm{~K}_{\sigma \pi}$, where $\mathrm{K}_{\sigma \pi}$ is the exchange integral in electronelectron repulsion, ${ }^{[25]}$ which is more consistent with the multireference results in Figure $2 \mathrm{~B}$.

The ground and excited spin state energetic comparisons were extended to the IPC analogs. Both DFT (Figure S3 and S7) and QD-NEVPT2 (Figure 3) were used to investigate the energetic dependencies on the dihedral angle. Several axial ligands were studied: $N$-methylimidazole $(N-$ MeImid), methyl thiolate, and hydroxide. DFT relaxed PESs are consistent with existing literature in that the CSS and OSS are either close in energy or the OSS is lowest (Figure S3 and S7). ${ }^{[8,20,21]}$ For all ligands, the amount of Hartree-Fock exchange has significant effects on the relative energies of the lowest triplet, CSS, and OSS. Note the lowest triplet in DFT is an Fe(III) coupled to a carbene anion radical (Table S30), which is different than the multireference description described below. Increasing Hartree-Fock exchange from 0 to $20 \%$ stabilizes the triplet relative to the OSS and CSS, as expected. While the CSS/OSS energies are virtually identical with BP86, the OSS is stabilized relative to the CSS with increasing Hartree-Fock exchange. Also, going from $N$ MeImid to thiolate to hydroxide, the triplet and the OSS states are stabilized in energy relative to the CSS. This is consistent with the increased stabilization of the formal Fe(III) redox level for these states. Thus, for all levels of theory and different axial ligands, DFT provides an OSS ground state description, and the energy of the OSS is stabilized further relative to the CSS with anionic ligands and larger amounts of Hartree-Fock exchange.

XAS and Mössbauer spectroscopies on IPC model complexes have been interpreted as reflecting an $\mathrm{Fe}$ (II) CSS ground state. ${ }^{[19]}$ DFT calculations were also performed on the synthetic 
complex [Fe(TPP)CCl $\mathrm{Cl}_{2}$ (tpp = tetraphenylporphyrin). Similar to other IPC complexes, the CSS and OSS states are nearly degenerate using the BP86, TPSSh, or B3LYP functionals (Table S4). The lowest energy triplet is $\sim 30 \mathrm{kcal} \mathrm{mol}^{-1}$ higher in energy and is also stabilized with increasing Hartree-Fock exchange. Thus, while the IPC analogs have low-lying CSS states, the presence of the OSS ground state description seems inconsistent with the available experimental data. These inconsistencies can be accounted for using multireference ab initio approaches.
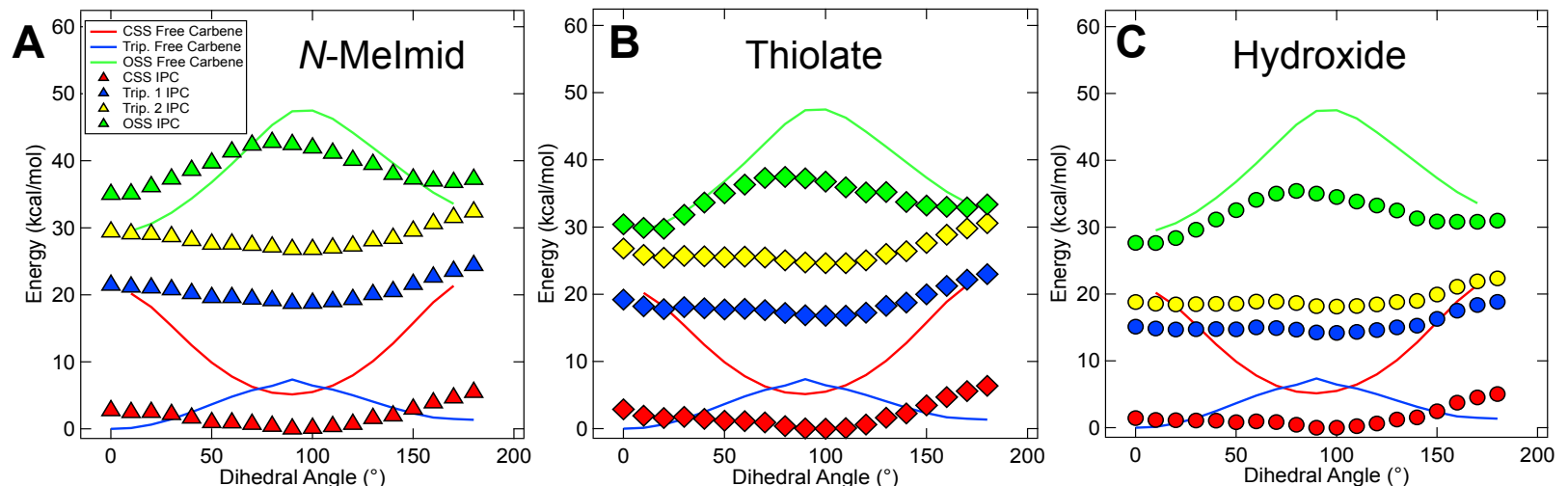

\begin{tabular}{|c|c|c|c|c|c|}
\hline $\begin{array}{cc}2 \\
4 & 2 \\
2 & 2 \\
\vdots & C O\end{array}$ & 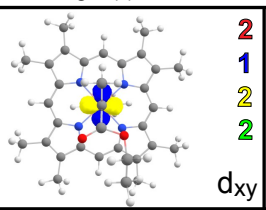 & 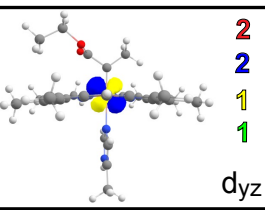 & 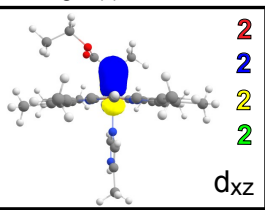 & 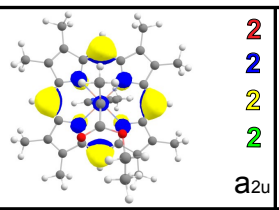 & 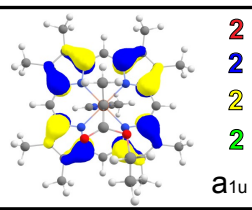 \\
\hline 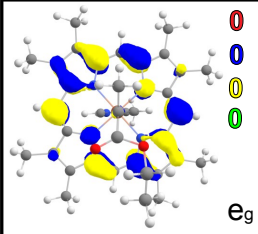 & 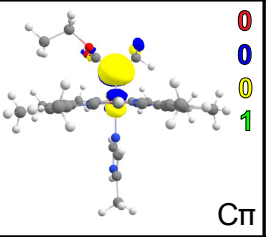 & $0^{0} a^{0} 0$ & 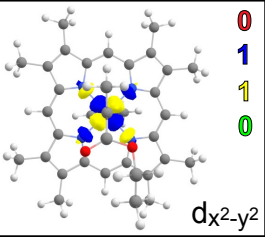 & $\therefore$ & "as. \\
\hline
\end{tabular}

Figure 3. (Top) Ground and excited state PESs along the dihedral coordinate calculated with quasi-degenerate $N$-electron valence second order perturbation theory (QD-NEVPT2) for (A) $N$ MeImid, (B) thiolate, and (C) hydroxide axial ligands. Free carbene QD-NEVPT2 results are depicted as solid lines. (Bottom) The 12 orbital active space for the $N$-MeImid IPC. Formal orbital occupation numbers are provided. The color of the number corresponds to the state given in the top panel (e.g., red and green numbers correspond to the CSS and OSS states, respectively). Note the triplets for the IPC (blue and yellow markers) are metal-based spin-forbidden ligand field transitions (i.e., no carbene radical and more CSS-like), while the triplet of the free carbene (blue lines) is necessarily carbene based.

The QD-NEVPT2 calculated PESs are given in Figures 3A-C for $N$-MeImid, thiolate, and hydroxide axial ligands, respectively. The CSS (red markers and numbers in Figure 3, top, bottom, respectively) is now the ground state for all angles and axial ligands (Tables S10, S15, S20). A global energy minimum exists at $\sim 90^{\circ}$, although the profile is shallow. The energetic minimum 
and angular dependence of the CSS state of the free carbene (red markers in Figure 2B and S1) are similar to those of the CSS state of the IPC (Figure 3 and S2). Also, as with the free carbene, the $360^{\circ}$ angular dependence of the IPC energetics are a mirror image about $180^{\circ}$ (Figure S2). This preference for the rotated conformation of the IPC is also consistent with the $\sim 266^{\circ}$ angle found in the active site of the carbene transferase enzyme. ${ }^{[8]}$ However, it is especially interesting to note that, while similar energies are observed here for the $\sim 90^{\circ}$ and $\sim 270^{\circ}$ IPC structures in the absence of additional interactions (Figure S2), only the latter conformation is observed in the protein structure, which suggests second sphere interactions play an important role in stabilizing one or the other conformation, as discussed by Lewis et al. ${ }^{[8]}$ Overall, this CSS ground state from multireference $a b$ initio methods is now more consistent with experimental data. ${ }^{[8,17-19]}$

In the multireference description, there are two triplet excited states between the CSS and OSS states (Figure 3, top), neither of which are carbene-based. Rather, these states are metal-based spin-forbidden ligand field transitions between filled Fe $d(x y) / d(y z)$ and unoccupied $d\left(x^{2}-y^{2}\right)$ orbitals (Figure 3, bottom). This is reflected by their formal occupation numbers given in Figure 3, bottom (blue and yellow numbers). Thus, given these triplet states are still formal Fe(II) configurations, their energy profiles in Figure 3 more closely resemble those of the CSS. In each case, the OSS is the highest energy state considered here, and it results from a MLCT transition between the Fe d(yz) and carbene $\mathrm{C} \pi$ orbitals, as depicted in Figure 3, bottom. Future optical and X-ray spectroscopic studies directed at characterizing the excited state ligand field and MLCT manifolds will be insightful.

While the CSS ground state of the IPCs is more consistent with experiment, the OSS could become important if structural distortions favoring the OSS occur along reaction coordinates. Indeed, the spin state energetics and ground state characters evolve significantly with $\mathrm{Fe}-\mathrm{C}$ bond elongation (Figure 4), an important coordinate to consider for reactivity. Only QD-NEVPT2 PESs are considered here, and the DFT results are given in Figure S6. While the CSS is the ground state in the QD-NEVPT2 calculated Fe-C PESs for all axial ligands, the energy of the OSS approaches the CSS state as the $\mathrm{Fe}-\mathrm{C}$ bond is elongated (compare red and green markers in Figures 4A-C). Furthermore, from analyses of the multireference ground state wave function (i.e., the configuration interaction (CI) vector), the amount of CSS character (red markers in Figures 4D$\mathrm{F}$ ), which is dominant at the $\mathrm{Fe}-\mathrm{C}$ equilibrium distance, decreases upon elongation of the $\mathrm{Fe}-\mathrm{C}$ bond. This decrease occurs with an increase in the OSS contribution (Table S24-6 and green 
markers in Figures 4D-F). For $N$-MeImid, the OSS character exceeds that of the CSS character at an $\mathrm{Fe}-\mathrm{C}$ distance of $\sim 2.3 \AA$. For the thiolate and hydroxide, this crossing occurs even later at $\sim 2.4$ and $2.5 \AA$, respectively. Overall, these bond distances are well within those of relevant transition states. ${ }^{[8,20]}$ These results suggest the OSS/CSS state mixing will be important to consider for understanding axial ligand dependent IPC reaction coordinates, which can translate to variations in selectivity and efficiency. ${ }^{[8]}$ For instance, transition state structures with elongated $\mathrm{Fe}-\mathrm{C}$ bonds may not be well described using DFT, and CI-vector analyses of ground state wave functions and related energies will be important for properly describing reaction mechanisms.
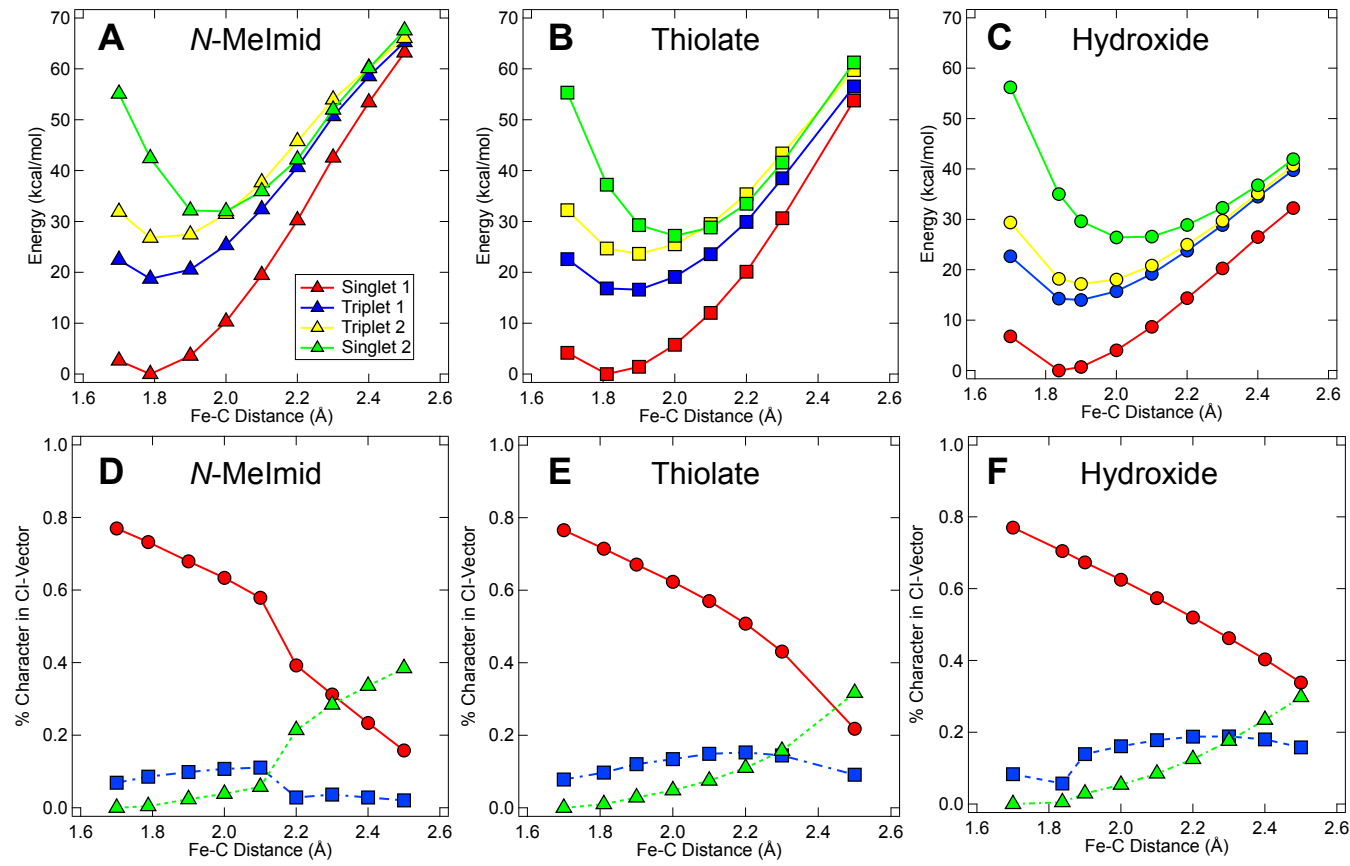

Figure 4. QD-NEVPT2 PESs along the Fe-carbene coordinate (top) and associated CI-vectors of the ground state (bottom): (A, D) N-MeImid, (B, E) thiolate, and (C, F) hydroxide. Red, CSS; green, OSS; and blue, the first triplet state.

In summary, discrepancies between DFT and experiment have hampered our understanding of IPC electronic structure. DFT predicts OSS ground states, while experimental data appear to favor a CSS description. Here we have shown that multireference methods provide a CSS ground state (i.e., electrophilic carbene) for all carbene conformations and axial ligands investigated. The OSS is also predicted to correspond to a high energy MLCT excited state (i.e., radical carbene) that is sensitive to the nature of the axial ligand. While the ground state is a CSS, the OSS character increases significantly upon elongating the $\mathrm{Fe}-\mathrm{C}$ bond. These changes in 
CSS/OSS characters will be important to consider for the reactivity and selectivity patterns of IPCs. Future studies should evaluate these contributions to better understand transition metal carbene catalysis.

\section{Acknowledgments.}

We acknowledge Dr. Ondřej Demel for use of the DLPNO-MR-CCSD program within the ORCA package. We thank Dr. Daniel Bím, Dr. Alec H. Follmer, and Mr. Ryan D. Ribson for helpful discussions. GDS thanks Dr. Mauricio Maldonado-Domínguez for guidance during early calculations. RGH gratefully acknowledges financial support from Caltech and the Dow Next Generation Educator Fund.

\section{References.}

[1] S. B. J. Kan, X. Huang, Y. Gumulya, K. Chen, F. H. Arnold, Nature 2017, 552, 132-136.

[2] K. Chen, X. Huang, S.-Q. Zhang, A. Z. Zhou, S. B. J. Kan, X. Hong, F. H. Arnold, Synlett 2019, 30, 378-382.

[3] H. M. Mbuvi, L. K. Woo, Organometallics 2008, 27, 637-645.

[4] Z. J. Wang, N. E. Peck, H. Renata, F. H. Arnold, Chem. Sci. 2013, 5, 598-601.

[5] G. Sreenilayam, R. Fasan, Chem. Commun. 2015, 51, 1532-1534.

[6] H. M. Mbuvi, E. R. Klobukowski, G. M. Roberts, L. K. Woo, J. Porphyr. Phthalocyanines 2010, 14, 284-292.

[7] S. B. J. Kan, R. D. Lewis, K. Chen, F. H. Arnold, Science 2016, 354, 1048-1051.

[8] R. D. Lewis, M. Garcia-Borràs, M. J. Chalkley, A. R. Buller, K. N. Houk, S. B. J. Kan, F. H. Arnold, Proc. Natl. Acad. Sci. 2018, 115, 7308-7313.

[9] V. Tyagi, R. B. Bonn, R. Fasan, Chem. Sci. 2015, 6, 2488-2494.

[10] K. Chen, S.-Q. Zhang, O. F. Brandenberg, X. Hong, F. H. Arnold, J. Am. Chem. Soc. 2018, 140, 16402-16407.

[11] P. S. Coelho, E. M. Brustad, A. Kannan, F. H. Arnold, Science 2013, 339, 307-310.

[12] M. Bordeaux, V. Tyagi, R. Fasan, Angew. Chem. Int. Ed. 2015, 54, 1744-1748.

[13] J. G. Gober, A. E. Rydeen, E. J. Gibson-O'Grady, J. B. Leuthaeuser, J. S. Fetrow, E. M. Brustad, ChemBioChem 2016, 17, 394-397.

[14] A. M. Knight, S. B. J. Kan, R. D. Lewis, O. F. Brandenberg, K. Chen, F. H. Arnold, ACS Cent. Sci. 2018, 4, 372-377.

[15] D. M. Carminati, R. Fasan, ACS Catal. 2019, 9, 9683-9697.

[16] M. J. Weissenborn, S. A. Löw, N. Borlinghaus, M. Kuhn, S. Kummer, F. Rami, B. Plietker, B. Hauer, ChemCatChem 2016, 8, 1636-1640.

[17] Y. Li, J.-S. Huang, Z.-Y. Zhou, C.-M. Che, X.-Z. You, J. Am. Chem. Soc. 2002, 124, 13185-13193.

[18] R. L. Khade, W. Fan, Y. Ling, L. Yang, E. Oldfield, Y. Zhang, Angew. Chem. Int. Ed. 2014, 53, 7574-7578. 
[19] Y. Liu, W. Xu, J. Zhang, W. Fuller, C. E. Schulz, J. Li, J. Am. Chem. Soc. 2017, 139, 5023-5026.

[20] D. A. Sharon, D. Mallick, B. Wang, S. Shaik, J. Am. Chem. Soc. 2016, 138, 9597-9610.

[21] H. Su, G. Ma, Y. Liu, Inorg. Chem. 2018, 57, 11738-11745.

[22] W. Kirmse, Carbene Chemistry, Academic Press, New York, 1971.

[23] F. Neese, Wiley Interdiscip. Rev. Comput. Mol. Sci. 2012, 2, 73-78.

[24] F. Neese, Wiley Interdiscip. Rev. Comput. Mol. Sci. 2018, 8, e1327.

[25] T. A. Albright, J. K. Burdett, M.-H. Whangbo, Orbital Interactions in Chemistry, WileyInterscience, Hoboken, New Jersey, 2013. 